\title{
Regimes de atmosfera controlada para o armazenamento de caqui 'Kyoto'
}

\author{
Controlled atmosphere conditions for 'Kyoto' persimmon storage
}

\author{
Auri Brackmann' ${ }^{1}$ Sérgio Tonetto de Freitas ${ }^{2}$ Ricardo Fabiano Hettwer Giehl ${ }^{3}$ \\ Anderson Machado de Mello $^{4}$ Marlova Benedetti ${ }^{5}$ \\ Viviani Ruffo de Oliveira ${ }^{6}$ Affonso José Wietzke Guarienti ${ }^{2}$
}

\begin{abstract}
O objetivo deste trabalho foi avaliar o efeito de regimes de atmosfera controlada associados ou não com o uso de baixa umidade relativa e com a aplicação pós-colheita de fungicida sobre a conservação da qualidade de caqui 'Kyoto'. Após o período de dois meses de armazenamento refrigerado a $-0,5^{\circ} \mathrm{C}$ mais cinco dias a $20^{\circ} \mathrm{C}$, a firmeza de polpa manteve-se mais elevada nos frutos submetidos a $0,5 \mathrm{kPa}$ de $\mathrm{O}_{2}$ e $5 \mathrm{kPa}$ de $\mathrm{CO}_{2}$. A maior incidência de podridões ocorreu nos frutos armazenados a $2 \mathrm{kPa}$ de $\mathrm{O}_{2}+10 \mathrm{kPa}$ de $\mathrm{CO}_{2}$. Baixa umidade relativa (90\%) ou pressões parciais elevadas de $\mathrm{CO}_{2}(10 \mathrm{a} 15 \mathrm{kPa})$ aumentaram o escurecimento da epiderme.
\end{abstract}

Palavras-chave: Diospyrus kaki L., qualidade pós-colheita, Alternaria alternata.

\section{ABSTRACT}

This work aimed to evaluate the effect of different partial pressures of $\mathrm{O}_{2}$ and $\mathrm{CO}_{2}$, combined or not with the use of low relative humidity $(\mathrm{RH})$ and the postharvest fungicide application, on the quality of 'Kyoto' persimmons during controlled atmosphere (CA) storage. After two months of storage at $-0.5^{\circ} \mathrm{C}$ plus five days at $20^{\circ} \mathrm{C}$, the highest flesh firmness was obtained in fruits stored in $\mathrm{CA}$ conditions of $0.5 \mathrm{kPa}$ of $\mathrm{O}_{2}$ and $5 \mathrm{kPa}$ of $\mathrm{CO}_{2}$. The highest rot incidence was observed in fruits stored at $2 \mathrm{kPaO}_{2}+10 \mathrm{kPaCO}$. Low $\mathrm{RH}(90 \%)$ or high $\mathrm{CO}_{2}$ levels (10 to $15 \mathrm{kPa}$ ) led to increased skin blackening.

Key words: Diospyrus kaki L., postharvest quality, Alternaria alternata.
O armazenamento de caquis em atmosfera controlada é uma prática cada vez mais freqüente. Segundo BRACKMANN \& SAQUET (1995), pressões parciais de $2 \mathrm{kPa}$ de $\mathrm{O}_{2}$ e $8 \mathrm{kPa}$ de $\mathrm{CO}_{2}$ são adequadas para o armazenamento de caquis 'Taubaté', 'Fuyu' e 'Bauru'. PRUSKY et al. (1997) observaram que a porcentagem de infecção de Alternaria alternata em frutos inoculados caiu de 90 para $1 \%$, quando o $\mathrm{CO}_{2}$ aumentou de 0 para $12 \mathrm{kPa}$ no interior de embalagens de polietileno. $\mathrm{O}$ uso do fungicida Iprodione em pré-colheita também reduziu significativamente o número de caquis cv. Triumph atacados por A. Alternata (PEREZ et al., 1995). Além disso, a infecção de $\boldsymbol{A}$. alternata em caquis é extremamente dependente de altos níveis de umidade relativa (PEREZ et al., 1995). Dados precisos em relação às melhores condições de armazenamento de caquis 'Kyoto' são escassos na literatura. Portanto, o objetivo deste trabalho foi avaliar o efeito de regimes de atmosfera controlada, associados ou não com o uso de baixa umidade relativa e com a aplicação póscolheita de fungicida, sobre a qualidade de caquis 'Kyoto' após o armazenamento.

O experimento foi conduzido no Núcleo de Pesquisa em Pós-Colheita (NPP) do Departamento de Fitotecnia da UFSM com frutos da cultivar Kyoto,

${ }^{1}$ Engenheiro Agrônomo, Doutor, Professor Adjunto, Departamento de Fitotecnia da Universidade Federal de Santa Maria (UFSM), 97105900, Santa Maria, RS. E-mail: brackman@ccr.ufsm.br. Autor para correspondência.

${ }^{2}$ Acadêmico do curso de Agronomia, UFSM. Bolsista do Programa Institucional de Bolsas de Iniciação Científica (PIBIC)/ Conselho Naional de Desenvolvimento Científico e Tecnológico (CNPq).

${ }^{3}$ Acadêmico do curso de Agronomia, UFSM. Bolsista Iniciação Científica da Fundação de Amparo à Pesquisa do Rio Grande do Sul.

${ }^{4}$ Engenheiro Agrônomo, mestrando do Programa de Pós-graduação em Agronomia (PPGA), UFSM. Bolsista do CNPq.

${ }^{5}$ Engenheiro Agrônomo, mestrando do PPGA, UFSM. Bolsista do CNPq.

${ }^{6}$ Nutricionista, mestrando do PPGA, UFSM. Bolsista do CNPq. 
provenientes de um pomar comercial da Serra Gaúcha RS. O delineamento experimental utilizado foi o inteiramente casualizado com quatro repetições e unidade experimental composta por 20 frutos. Os frutos foram armazenados $\mathrm{a}-0,5^{\circ} \mathrm{C}$, sendo que os tratamentos avaliados foram: regimes de atmosfera controlada (AC) com $2 \mathrm{kPa}$ de $\mathrm{O}_{2}+15 \mathrm{kPa}$ de $\mathrm{CO}_{2}$; $2 \mathrm{kPa}$ de $\mathrm{O}_{2}+10 \mathrm{kPa}$ de $\mathrm{CO}_{2} ; 1 \mathrm{kPa}$ de $\mathrm{O}_{2}+8 \mathrm{kPa}$ de $\mathrm{CO}_{2} ; 0,5 \mathrm{kPa}$ de $\mathrm{O}_{2}+5 \mathrm{kPa}$ de $\mathrm{CO}_{2} ;$ e $1 \mathrm{kPa}$ de $\mathrm{O}_{2}+$ $5 \mathrm{kPa}$ de $\mathrm{CO}_{2}$, sendo esta última condição associada com baixa umidade relativa $(90 \%)$ e com o uso de

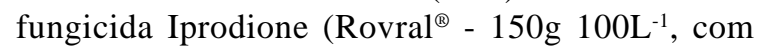
imersão dos frutos por três minutos). Nos demais regimes de AC, foi utilizada umidade relativa de $96 \%$. Após dois meses de armazenamento e mais cinco dias de exposição a $20^{\circ} \mathrm{C}$, foram realizadas as análises físico-químicas, quando se avaliou a firmeza de polpa, sólidos solúveis totais (SST) e incidência de podridões, conforme descrito por BRACKMANN et al. (1997); escurecimento da epiderme, em uma escala de 0 a 3, onde $0=$ sem escurecimento, $1=$ até $20 \%$ da epiderme escurecida, $2=$ de 21 a $50 \%$ da epiderme escurecida e $3=$ mais de $50 \%$ da epiderme escurecida; cor da epiderme, obtida por um colorímetro eletrônico, da marca Minolta, modelo CR-310, sendo os resultados expressos através dos valores de $\mathrm{L}^{*}$ (brilho), $\mathrm{C}^{*}$ (croma) e $\mathrm{h}^{\circ}$ (ângulo hue). Os dados, expressos em porcentagem, foram transformados para arco cosseno da raiz quadrada de $\mathrm{x} / 100$ e submetidos à análise da variância. As médias foram comparadas entre si pelo teste de Duncan em nível de 5\% de probabilidade de erro.

$\mathrm{O}$ armazenamento em $\mathrm{AC}$ com $0,5 \mathrm{kPa}$ de $\mathrm{O}_{2}$ e $5 \mathrm{kPa}$ de $\mathrm{CO}_{2}$ manteve a firmeza de polpa mais elevada (Tabela 1). No entanto, esta diferença foi pouco expressiva em relação aos demais tratamentos. Os sólidos solúveis totais (SST) apresentaram-se mais elevados no tratamento com baixa umidade relativa (Tabela1), o que ocorreu, provavelmente, devido à perda de água e ao aumento da concentração de açúcares nos frutos. Os tratamentos com $1 \mathrm{kPa}$ de $\mathrm{O}_{2}$ e $5 \mathrm{kPa}$ de $\mathrm{CO}_{2}$ mais aplicação de fungicida Iprodione ou baixa umidade relativa (90\%) apresentaram os menores valores absolutos de incidências de frutos podres (Tabela 1). Este resultado está de acordo com PEREZ et al. (1995), que obtiveram menor incidência de podridão em caquis tratados com Iprodione. $\mathrm{O}$ escurecimento da epiderme foi menor nos tratamentos com $1 \mathrm{kPa}$ de $\mathrm{O}_{2}$ combinado com 5 ou $8 \mathrm{kPa}$ de $\mathrm{CO}_{2}$ (Tabela 1). De acordo com BENARIE \& OR (1986), baixas pressões de $\mathrm{O}_{2}$ parecem estar envolvidas com a inibição da atividade da enzima polifenol oxidase (PPO), responsável pela formação de pigmentos escuros na epiderme. Em relação à cor da epiderme, não se observou diferença significativa nos valores de L* (Tabela 1). No entanto, os frutos submetidos a

Tabela 1 - Qualidades físico-químicas de caqui 'Kyoto' armazenado a $-0,5^{\circ} \mathrm{C}$ por dois meses em atmosfera controlada seguido de cinco dias a $20^{\circ}$ C. Santa Maria, RS, 2001.

\begin{tabular}{|c|c|c|c|c|c|c|c|}
\hline \multirow{2}{*}{$\begin{array}{l}\text { Condição de armazenamento } \\
\left.\left(\mathrm{kPa} \mathrm{O}_{2}+\mathrm{kPa} \mathrm{CO}\right)_{2}\right)\end{array}$} & \multirow{2}{*}{$\begin{array}{l}\text { Firmeza de polpa } \\
\text { (N) }\end{array}$} & \multirow{2}{*}{$\begin{array}{c}\text { SST } \\
\left({ }^{\circ} \text { Brix }\right)\end{array}$} & \multirow{2}{*}{$\begin{array}{l}\text { Podridão } \\
\quad(\%)\end{array}$} & \multirow{2}{*}{$\begin{array}{c}\text { Escurecimento } \\
\text { da epiderme } \\
(0-3)^{1}\end{array}$} & \multicolumn{3}{|c|}{ Cor $^{2}$} \\
\hline & & & & & $\mathrm{L}^{*}$ & $\mathrm{C}^{*}$ & $\mathrm{~h}^{\circ}$ \\
\hline $2+15$ & $6,88{a b^{3}}^{3}$ & $16,3 \mathrm{ab}$ & $46,0 \mathrm{~b}$ & $3,00 \mathrm{a}$ & $59,6 \mathrm{a}$ & $63,6 \mathrm{a}$ & $64,8 \mathrm{a}$ \\
\hline $2+10$ & $6,17 \mathrm{abc}$ & $15,9 \mathrm{bc}$ & 59,9 a & $3,00 \mathrm{a}$ & $47,5 \mathrm{a}$ & $43,5 \mathrm{~b}$ & $48,4 b$ \\
\hline $1+8$ & $5,68 \mathrm{bc}$ & $15,9 \mathrm{bc}$ & $38,2 \mathrm{~b}$ & $2,02 \mathrm{c}$ & $60,5 \mathrm{a}$ & $59,3 \mathrm{ab}$ & $66,2 \mathrm{a}$ \\
\hline $1+5$ & $5,91 \mathrm{abc}$ & $15,4 \mathrm{~d}$ & $46,1 \mathrm{~b}$ & $1,94 \mathrm{c}$ & $57,1 \mathrm{a}$ & $59,7 \mathrm{ab}$ & $63,7 \mathrm{a}$ \\
\hline $\begin{array}{l}1+5 \text { e Baixa umidade } \\
\text { relativa }\end{array}$ & $6,79 a b$ & $16,6 \mathrm{a}$ & $25,5 \mathrm{~b}$ & $2,58 \mathrm{~b}$ & 58,5 a & $61,8 \mathrm{ab}$ & $64,7 \mathrm{a}$ \\
\hline $1+5$ e Fungicida (Iprodione) & $5,55 \mathrm{c}$ & $16,0 \mathrm{bc}$ & $23,5 \mathrm{~b}$ & $1,76 \mathrm{c}$ & $57,7 \mathrm{a}$ & $60,2 \mathrm{ab}$ & $64,4 \mathrm{a}$ \\
\hline $0,5+5$ & $6,97 \mathrm{a}$ & $15,6 \mathrm{~cd}$ & $43,0 \mathrm{~b}$ & $2,44 \mathrm{~b}$ & $54,3 \mathrm{a}$ & $54,0 \mathrm{ab}$ & $63,9 \mathrm{a}$ \\
\hline $\mathrm{CV}(\%)$ & 11,5 & 1,78 & 32,3 & 12,5 & 19,85 & 20,05 & 19,67 \\
\hline
\end{tabular}

${ }^{1}$ Valores expressos em índices, onde índice $0=$ sem escurecimento, $1=$ até $20 \%$ da epiderme escurecida, $2=$ de 21 a $50 \%$ e $3=$ mais de $50 \%$ da epiderme escurecida.

${ }^{2} \mathrm{~L}^{*}=$ brilho; $\mathrm{C}^{*}=$ croma; e $\mathrm{h}^{\circ}=$ ângulo hue, onde $0^{\circ}$ corresponde à coloração vermelho-púrpura, $90^{\circ}$ à coloração amarela, $180^{\circ}$ verdeazulado e $270^{\circ}$ à cor azul.

${ }^{3}$ Tratamentos com médias não seguidas pela mesma letra na vertical diferem estatisticamente entre si, pelo teste de Duncan em nível de 5\% de probabilidade de erro.

Ciência Rural, v.34, n.6, set-out, 2004. 
$2 \mathrm{kPa}$ de $\mathrm{O}_{2}+10 \mathrm{kPa}$ de $\mathrm{CO}_{2}$ apresentavam-se com os menores valores de $\mathrm{C}^{*}$ e com coloração da epiderme mais vermelha.

$\mathrm{O}$ regime de atmosfera controlada com $1 \mathrm{kPa}$ de $\mathrm{O}_{2}$ e $5 \mathrm{kPa}$ de $\mathrm{CO}_{2}$, associado ou não com a aplicação de fungicida (Iprodione) em pós-colheita, foi o mais adequado para a manutenção da qualidade pós-colheita de caqui 'Kyoto'. O uso de pressões parciais de $\mathrm{CO}_{2}$ elevadas (10 a $15 \mathrm{kPa})$ e de baixa umidade relativa promoveram um aumento do escurecimento da epiderme.

\section{REFERÊNCIAS BIBLIOFRÁFICAS}

BEN-ARIE, R.; OR, E. The development and control of husk scald on 'Wonderful' pomegranate fruit during storage. Journal of American Society for Horticultural Science, Alexandria, v.11, p.395-399, 1986.
BRACKMANN, A.; MAZARO, S.M.; SAQUET, A.A. Frigoconservação de caquis (Diospyrus kaki, L.) das cultivares Fuyu e Rama Forte. Ciência Rural, Santa Maria, v.27, n.4, p.561-565, 1997.

BRACKMANN, A.; SAQUET, A.A. Efeito da temperatura e condições de atmosfera controlada sobre a conservação de caqui (Diospyrus kaki L.). Ciência Rural, Santa Maria, v.25, n.2, p.215-218, 1995.

PEREZ, A.; BEN-ARIE, R.; DINOOR, A. Prevention of black spot disease in persimmon fruit by gibberellic acid and iprodione treatments. Phytopathology, Palo Alto, v.85, n.2, p.221-225, 1995 .

PRUSKY, D.; PEREZ, A.; ZUTKHI, Y. Effect of modified atmosphere for control of black spot, caused by Alternaria alternata, on stored persimmon fruits. Phytopathology, Palo Alto, v.87, n.2, p.203-208, 1997 . 